\title{
Encephaloduroarteriosynangiosis for pediatric moyamoya disease: long-term follow-up of 100 cases at a single center
}

\author{
*Yong Zhang, MD, MS, ${ }^{1,2}$ Xiang-Yang Bao, MD, MS, ${ }^{2}$ Lian Duan, MD, PhD, ${ }^{1,2}$ \\ Wei-Zhong Yang, MD, MS, ${ }^{2}$ De-Sheng Li, MD, PhD, ${ }^{2}$ Zheng-Shan Zhang, MD, MS, ${ }^{2}$ \\ Cong Han, MD, MS, ${ }^{2}$ Feng Zhao, MD, MS, ${ }^{2}$ Qian Zhang, MD, MS, ${ }^{2}$ and Qian-Nan Wang, MD, MS ${ }^{2}$

\begin{abstract}
1'Department of Neurosurgery, 307th Hospital of People's Liberation Army, 307 Clinical College, Anhui Medical University; and 2Department of Neurosurgery, 307th Hospital of People's Liberation Army, The Center for Cerebral Vascular Disease, PLA, Beijing, People's Republic of China
\end{abstract}

OBJECTIVE The object of this study was to summarize the long-term effect of encephaloduroarteriosynangiosis (EDAS) for the treatment of pediatric moyamoya disease (MMD) and to investigate factors influencing the clinical outcomes of EDAS.

METHODS Clinical features, angiographic findings, and clinical outcomes were analyzed among MMD patients younger than 18 years who had been treated with EDAS between 2002 and 2007 at the authors' institution. The Kaplan-Meier method was used to estimate stroke risk after EDAS. Predictors of neurological outcome were assessed.

RESULTS One hundred fifteen patients were identified. The mean age at symptom onset was $7.3 \pm 4.0$ years. The incidence of familial MMD was $11.3 \%$. The female/male ratio was 1:1.16. A total of 232 EDAS procedures were performed, and the incidence of postoperative complications was $3 \%$. Postoperative digital subtraction angiography was performed in $54 \%$ of the patients, and about $80 \%$ of the hemispheres showed good or excellent results. Neovascularization showed significant correlations with delay time (from symptom onset to first operation), Suzuki stage, and preoperative stroke (all $p<0.05$ ). Clinical follow-up was available in 100 patients with a mean follow-up of $124.4 \pm 10.5$ months. Ten-year cumulative survival was $96.5 \%$ after surgery, and the risk of stroke was $0.33 \%$ /person-year. An independent life with no significant disability was reported by $92 \%$ of the patients. A good outcome correlated with a low Suzuki stage $(p=0.001)$. Older children and those without preoperative stroke had better clinical outcomes $(p<0.05)$.

CONCLUSIONS On the basis of long-term follow-up data, the authors concluded that EDAS is a safe and effective treatment for pediatric MMD, can reduce the risk of subsequent neurological events, and can improve quality of life. The risk of ischemia-related complications was higher in younger patients, and older children showed better outcomes. Compensation was greater with more prominent cerebral ischemia. The long-term clinical outcome largely depended on the presence and extent of preoperative stroke.

https://thejns.org/doi/abs/10.3171/2018.2.PEDS17591

KEYWORDS moyamoya disease; encephaloduroarteriosynangiosis; pediatric; long-term outcome; vascular disorders

$\mathrm{M}$ OYAMOYA disease (MMD) is a cerebrovascular disorder in which vessel stenosis or occlusion progresses from the internal carotid artery to the anterior, middle, and posterior cerebral arteries and dilated collateral vessels appear at the base of the brain. ${ }^{40}$ Children are at high risk for MMD with recurrent cerebral ischemic attacks, and indirect revascularization is an effective treatment for symptomatic pediatric MMD. ${ }^{4,11,12,15}$

Encephaloduroarteriosynangiosis (EDAS), an indirect bypass method for pediatric MMD, was first reported in 1981 by Matsushima et al. ${ }^{35}$ Generally, EDAS involves placement of an external carotid artery branch beneath the

ABBREVIATIONS CBF = cerebral blood flow; EDAS = encephaloduroarteriosynangiosis; $M C A=$ middle cerebral artery; $M M D=$ moyamoya disease; MRA = MR angiography; $\mathrm{mRS}=$ modified Rankin Scale; PCA = posterior cerebral artery; STA = superficial temporal artery; TIA = transient ischemic attack.

SUBMITTED October 28, 2017. ACCEPTED February 27, 2018.

INCLUDE WHEN CITING Published online June 1, 2018; DOI: 10.3171/2018.2.PEDS17591.

* Y.Z. and X.Y.B. contributed equally to this work and share first authorship. 
dura mater in ischemic territories ${ }^{35}$ MMD is rapidly progressive in children, and long-term follow-up is a more objective index of the effect of surgical treatment; however, there are few long-term, large-sample follow-up studies of EDAS for pediatric MMD.

Since 2002, we have surgically treated more than 1000 patients with pediatric MMD, and EDAS has been the preferred surgical revascularization procedure at our institute. Thus, we performed a retrospective review of our clinical records and outcomes. In this study, we primarily investigated the long-term efficacy of EDAS for pediatric MMD.

\section{Methods \\ Patient Selection}

We initially selected the records of all patients younger than 18 years who had been treated using EDAS between 2002 and 2007 at the Department of Neurosurgery, 307th Hospital of People's Liberation Army, Beijing. The hospital research ethics board approved this study. All patients had been diagnosed with MMD according to current criteria, as follows. Cerebral angiography or MR angiography (MRA) must at least show 1) stenosis or occlusion of the terminal portion of the intracranial internal carotid artery or proximal portions of the anterior and/or middle cerebral artery (MCA), 2) abnormal vascular networks in the vicinity of the occlusive or stenotic lesions in the arterial phase, and 3) bilaterality of findings 1 and 2 . We included 10 patients with unilateral MMD in the study. Patients with secondary moyamoya phenomenon caused by meningitis, Down syndrome, systemic vasculitis, hyperthyroidism, neurofibromatosis, leptospirosis infection, or previous skull base radiation therapy were not included in our study.

\section{Retrospective Chart Review}

We reviewed hospital charts and clinic notes for information on patient sex, age at symptom onset and surgery, family history, presence of an aneurysm, initial modified Rankin Scale (mRS) score, surgical revascularization, and radiological data. The preoperative angiographic stage was evaluated according to Suzuki's classification. The higher Suzuki stage was used when the 2 hemispheres had different findings ${ }^{40}$ The development of collateral circulation of the MCA through the bypass was graded according to a system described by Matsushima et al.: ${ }^{34} 1$ ) excellent indicates that the revascularization area involves more than two-thirds of the MCA distribution, 2) good indicates one-third to two-thirds of the MCA territory, 3) fair indicates less than one-third of the MCA distribution, and 4) none indicates no collateral circulation. All radiographic results were evaluated by 2 doctors with more than 5 years of experience.

\section{Surgical Treatment}

All patients underwent EDAS performed by a single surgeon. At our institute, the indications for surgery include 1) diagnosed MMD or unilateral MMD based on angiography or MRA, and 2) clinical symptoms due to apparent cerebral ischemia or decreased regional cerebral blood flow (CBF). In bilateral MMD, the clinically more symptomatic side (the hemisphere with frequent transient ischemic attacks [TIAs]) or the dominant side is revascularized first. Generally, in the absence of complications, the other side is revascularized 3 months after the first surgery.

The key step is to take a scalp artery with a strip of galea, leaving the distal as well as the proximal arteries intact, and transplant it to a narrow linear dural opening made under an osteoplastic craniotomy, as described by Matsushima et al. ${ }^{35}$ Specifically, the skin is incised over the superficial temporal artery (STA), and the artery is meticulously dissected from surrounding tissue and underlying temporalis fascia and muscle. Two bone holes are made beneath the proximal and distal ends of the arterial bridge. Bone holes are connected to make an oval bone flap (average size $3.0 \times 8.0 \mathrm{~cm}$ ), the dura mater is opened, and the STA is sutured to the exposed dural interface. The bone flap is replaced with ample space for the vessel. The STA is most commonly used, but the occipital artery can also be used if there is posterior cerebral artery (PCA) involvement.

All patients are given a glucose-saline infusion according to guidelines for pediatric hydration the day before and after surgery. During surgery, partial pressure of $\mathrm{CO}_{2}\left(\mathrm{PaCO}_{2}\right)$ levels are strictly maintained between 33 and $36 \mathrm{~mm} \mathrm{Hg}$, and it is necessary to reduce the risk of acute ischemic stroke. The mean arterial systolic pressure is maintained between 100 and $120 \mathrm{~mm} \mathrm{Hg}$. Postoperative analgesia is provided for pediatric patients. . $^{10,27,42}$ To control postoperative pain, an intermittent injection of dexmedetomidine hydrochloride $(0.5 \mu \mathrm{g} / \mathrm{kg} / \mathrm{hr})$ is used. When a new cerebral infarction occurs without epidural or subdural hemorrhage, neuroprotective agents, mild elevation of blood pressure, proper dehydration therapy, mask $\mathrm{O}_{2}$ supplementation, bed rest, and hyperbaric $\mathrm{O}_{2}$ can be used. Evacuation of an epidural or subdural hematoma is necessary if there is evidence of brain compression, to prevent further ischemic events.

\section{Angiography Follow-Up}

We recommended that each patient undergo cerebral arteriography at 6 months after surgery to assess the efficacy of synangiosis and guide subsequent treatment. However, this recommendation was refused by some families or could not be completed in other cases because of economic or other considerations.

\section{Clinical Follow-Up}

After discharging patients, we evaluated long-term outcomes with clinic visits and telephone or mailed interviews. All work was performed by an experienced doctor, according to uniform protocols. Predictors of neurological outcome were assessed, and the mRS was used to determine functional neurological outcomes. ${ }^{5}$ The overall clinical outcomes were divided into 4 categories: 1) excellent, complete resolution of preoperative symptoms (such as TIA or seizures) and no fixed neurological deficits; 2) good, complete resolution of symptoms but with mild residual neurological deficits; 3) fair, persistent but less frequent symptoms; and 4) poor, symptoms unchanged 
or worsened. For patients who could not be contacted or lacked data, clinical features and long-term outcomes at the last clinic visit were used. ${ }^{6}$ The Kaplan-Meier method was used to estimate stroke risk after EDAS.

Postoperative stroke was defined as a new neurological deficit lasting 24 hours or more after revascularization surgery, in association with a new infarct or hemorrhage on MRI or CT in the first 30 days after the revascularization procedure. ${ }^{6}$

\section{Statistical Analysis}

We classified patient age and delay time into 4 groups as categorical variables according to the quartile, used the chi-square test (including all characteristics) and multivariate regression analysis (including symptom onset age, delay time, preoperative stroke, and Suzuki stage) for correlations between patient characteristics and the degree of neovascularization or clinical outcomes of EDAS, and then used the Student t-test to analyze the difference in the mRS score before and after EDAS. The annual risk of stroke was calculated with a person-year method, and cumulative survival was evaluated using the Kaplan-Meier method. A p value $<0.05$ was considered statistically significant. Data were analyzed using SPSS for Windows, version 19.0 (SPSS Inc.).

\section{Results}

\section{Demographics and Clinical Presentation}

We identified 115 pediatric MMD patients who had been treated between January 2002 and December 2007. The mean ( \pm standard deviation) age at symptom onset was $7.3 \pm 4.0$ years (range 1.21-17.37 years). There were 53 girls and 62 boys. The incidence of familial MMD was $11.3 \%(13 / 115)$.

Initial symptoms were TIA (54.78\%), infarction (13.91\%), or headache $(26.96 \%)$. Angiographic Suzuki stages were 1 (3.48\%), 2 (8.70\%), $3(27.83 \%), 4(25.22 \%), 5$ (26.96\%), and $6(7.83 \%) ; 10(8.70 \%)$ patients had unilateral disease. Neurological deficits were present before surgery in 29 cases, and 9 patients had disability (mRS score $>2$; Table 1).

\section{Surgical Treatment}

Of 232 EDAS procedures, 94 cases were bilateral, and the occipital artery was used in 23 . The mean age at the first operation was $10.1 \pm 3.7$ years (range 2.3-17.9 years), and the mean time from symptom onset to the first operation (delay time) was $2.82 \pm 2.96$ years. The delay in more than $60 \%$ of the patients was longer than 1 year, and $73.9 \%$ of patients were initially misdiagnosed and treated incorrectly; only a quarter of the patients underwent surgery within 6 months. Postoperative complications occurred in $7(3.01 \%)$ cases, with cerebral infarction in 3, TIA in 1, epidural hematoma in 1, and subdural hematoma in 2. Two of the cerebral infarctions occurred in patients who were younger than 3 years and cried loudly in the first 24 hours after surgery. Fortunately, 6 cases improved after individualized treatment, but the patients younger than 2 years had neurological deficits due to parietotemporal lobe infarction (mRS score 3-4).
TABLE 1. Clinical features of 115 pediatric patients with MMD

\begin{tabular}{lc}
\hline \multicolumn{1}{c}{ Characteristic } & Value \\
\hline Age at symptom onset in yrs (range) & $7.3 \pm 4.0(1.21-17.4)$ \\
\hline Age at first surgery in yrs (range) & $10.1 \pm 3.7(2.3-17.9)$ \\
\hline Delay time in yrs (range) $\dagger$ & $2.82 \pm 2.96(0.02-12.76)$ \\
\hline Misdiagnosed or improperly treated & $85(73.9 \%)$ \\
\hline Females & $53(46.1 \%)$ \\
\hline Familial occurrence & $13(11.3 \%)$ \\
\hline PCA involvement & $49(42.61 \%)$ \\
\hline Unilat lesions & $10(8.70 \%)$ \\
\hline Preop stroke & $29(25.22 \%)$ \\
\hline Initial symptoms & $63(54.78 \%)$ \\
\hline TIA & $31(26.96 \%)$ \\
\hline Headache & $16(13.91 \%)$ \\
\hline Infarction & $3(2.61 \%)$ \\
\hline Seizure & $1(0.87 \%)$ \\
\hline Hemorrhage & $1(0.87 \%)$ \\
\hline Asymptomatic & \\
\hline Angiographic Suzuki stage & $4(3.48 \%)$ \\
\hline 1 & $10(8.70 \%)$ \\
\hline 2 & $32(27.83 \%)$ \\
\hline 3 & $29(25.22 \%)$ \\
\hline 4 & $31(26.96 \%)$ \\
\hline Age at first operation. & $9(7.83 \%)$ \\
\hline MMD not diagnosed or only nonoperative treatment at initial diagnosis. \\
\hline $0-2$
\end{tabular}

\section{Follow-Up Digital Subtraction Angiography}

Digital subtraction angiography was performed after EDAS in 62 cases (54\%) involving 114 hemispheres (at a mean of $36.5 \pm 32.9$ months, range 4.13-129.93 months). Excellent neovascularization was achieved in 65 hemispheres $(57.02 \%)$, good in $26(22.81 \%)$, and fair in $20(17.54 \%)$. Three hemispheres $(2.63 \%)$ showed no neovascularization. There were significant correlations between neovascularization and delay time (chi-square $=$ $21.479, \mathrm{p}=0.028$ ), Suzuki stage (chi-square $=39.956, \mathrm{p}=$ 0.014 ), and preoperative stroke (chi-square $=8.052, \mathrm{p}=$ 0.036; Table 2). Multivariate regression analysis was used for meaningful factors, but the model for the relationships between correlated factors and neovascularization showed no statistical significance $(\mathrm{F}=1.186, \mathrm{p}=0.3276)$.

\section{Long-Term Clinical Outcomes}

Follow-up after surgery was performed on 115 patients in this series, but 15 (13\%) were lost to follow-up; therefore, data from 100 patients were used in our analy- 
TABLE 2. Analysis of factors predictive of collateral circulation and clinical outcomes

\begin{tabular}{|c|c|c|c|c|}
\hline \multirow[b]{2}{*}{ Characteristic } & \multicolumn{2}{|c|}{ Collateral Circulation } & \multicolumn{2}{|c|}{ Clinical Outcome } \\
\hline & $\chi^{2}$ & p Value & $\chi^{2}$ & $p$ Value \\
\hline Age at symptom onset & 4.049 & 0.399 & 19.777 & 0.001 \\
\hline Delay time & 21.479 & 0.028 & 1.1821 & 0.769 \\
\hline Age at surgery & 1.024 & 0.906 & 18.679 & 0.001 \\
\hline Initial symptoms & 2.308 & 0.511 & 5.663 & 0.129 \\
\hline Unilat lesions & 1.454 & 0.275 & 0.003 & 0.957 \\
\hline PCA involvement & 0.402 & 0.558 & 0.004 & 0.950 \\
\hline Suzuki stage & 39.956 & 0.014 & 23.470 & 0.001 \\
\hline Preop stroke & 8.052 & 0.036 & 8.464 & 0.004 \\
\hline Collateral circulation & & & 0.578 & 0.901 \\
\hline
\end{tabular}

Boldface type indicates statistical significance.

sis. The mean follow-up among these patients was 124.4 \pm 10.5 months (range 108.4-154.9 months), the median was 123.3 months, and $67 \%$ of the patients were followed up for more than 120 months. Ten-year cumulative survival was $96.5 \%$ after surgery. The overall final clinical outcome was excellent in $67 \%$, good in $25 \%$, fair in $4 \%$, and poor in $4 \%$. More than $90 \%$ of the children with TIA or headache improved significantly; prognoses in patients with various initial symptoms are shown in Fig. 1. Unfortunately, cerebrovascular events occurred in 4 patients: 7 years after bilateral surgery with excellent neovascularization, the patient in case 1 had basal ganglia hemorrhage

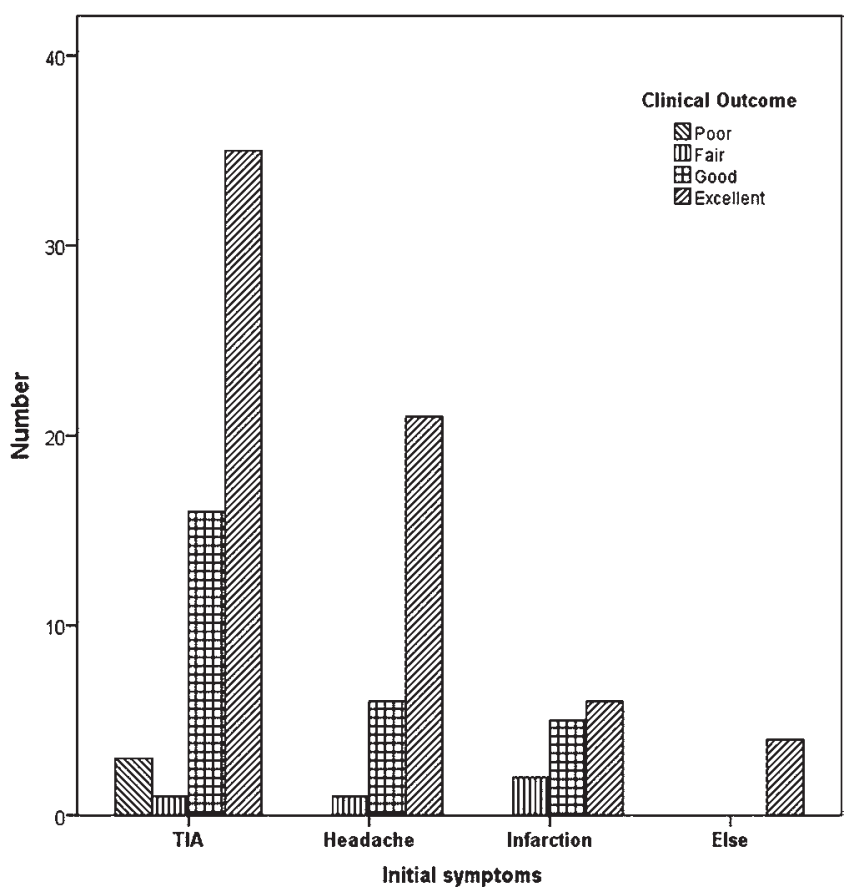

FIG. 1. Clinical outcomes for each symptom. There was a good prognosis in most patients with initial symptoms of TIA or headache. There were few differences in clinical outcomes according to the initial symptom, and there was no statistical significance in these differences.

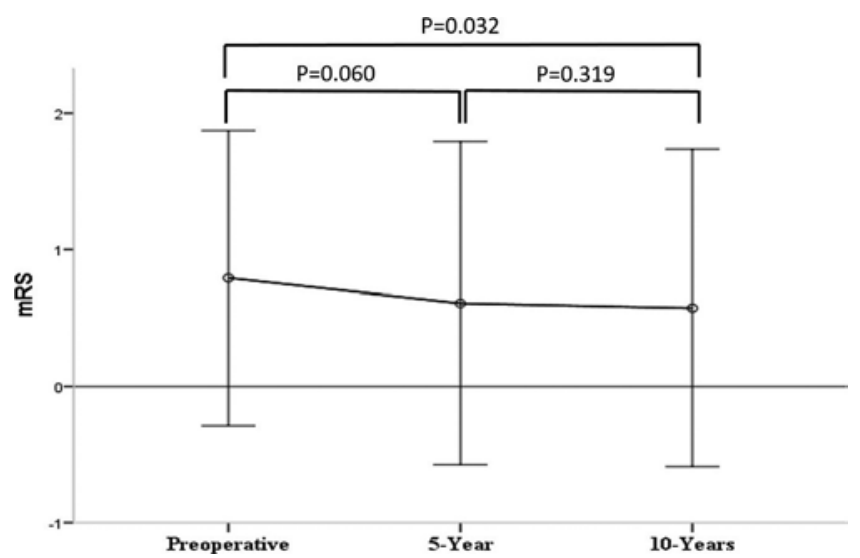

FIG. 2. Comparison of clinical status at each period scored using the $\mathrm{mRS}$.

and ventricular hemorrhage and died; about 2 years after bilateral surgery with good neovascularization, the patient in case 2 had a parietotemporooccipital lobe infarction; 3 years and 6 months after surgery, the patients in cases 3 and 4, respectively, had infarctions in the operated hemisphere. These latter 2 cases never returned for follow-up after infarction, and incomplete information was obtained by phone. Among 5 patients who had undergone surgery before the age of 3 years, 2 did not recover because of infarction in the first 24 hours postoperatively. Among the children older than 3 years, 1 had a poor outcome, 4 had fair outcomes, and all others $(91.3 \%)$ had a good or excellent outcome. In the group with symptom onset at an age younger than 3 years, 5 (26.3\%) of 19 had poor or fair outcomes, 9 had good outcomes, and 5 had excellent outcomes.

Based on the mRS, the mean preoperative clinical score for all patients was $0.79 \pm 1.08$ (range $0-4$ ); the mean short-term (about 5 years) and long-term (about 10 years) clinical scores after surgery were $0.61 \pm 1.18$ (range 0-6) and $0.57 \pm 1.16$ (range 0-6; Fig. 2). Among the patients, 28 were improved, 66 were unchanged, and 6 were worse, and $92 \%$ of the patients could live independently, with no or mild disability (mRS score $0-2)$. Six patients $(6 \%)$ had an mRS score of 3 or 4 , with moderate or severe disability, and 2 had an mRS score of 6. During the entire follow-up period, the rate of any stroke in all patients was $0.33 \%$ / person-year. The 5- and 10-year cumulative risks of any stroke were $2.6 \%$ and $3.5 \%$ for all patients treated using surgical revascularization (Fig. 3).

Analysis of preoperative clinical variables showed that an older age at symptom onset and at first surgery (chisquare $=19.777, \mathrm{p}=0.001 ;$ chi-square $=18.697, \mathrm{p}=0.001$, respectively) were associated with a more favorable clinical outcome. However, a higher Suzuki stage (chi-square = $23.470, \mathrm{p}=0.001$ ) and preoperative infarction (chi-square $=8.464, \mathrm{p}=0.004)$ were associated with a slightly worse clinical outcome. Correlations between outcomes and initial symptoms $(p=0.129)$, unilateral lesions $(p=0.957)$, or PCA involvement $(\mathrm{p}=0.950)$, as well as the grade of neovascularization ( $\mathrm{p}=0.901$; Table 2 ) showed no statistical significance. 


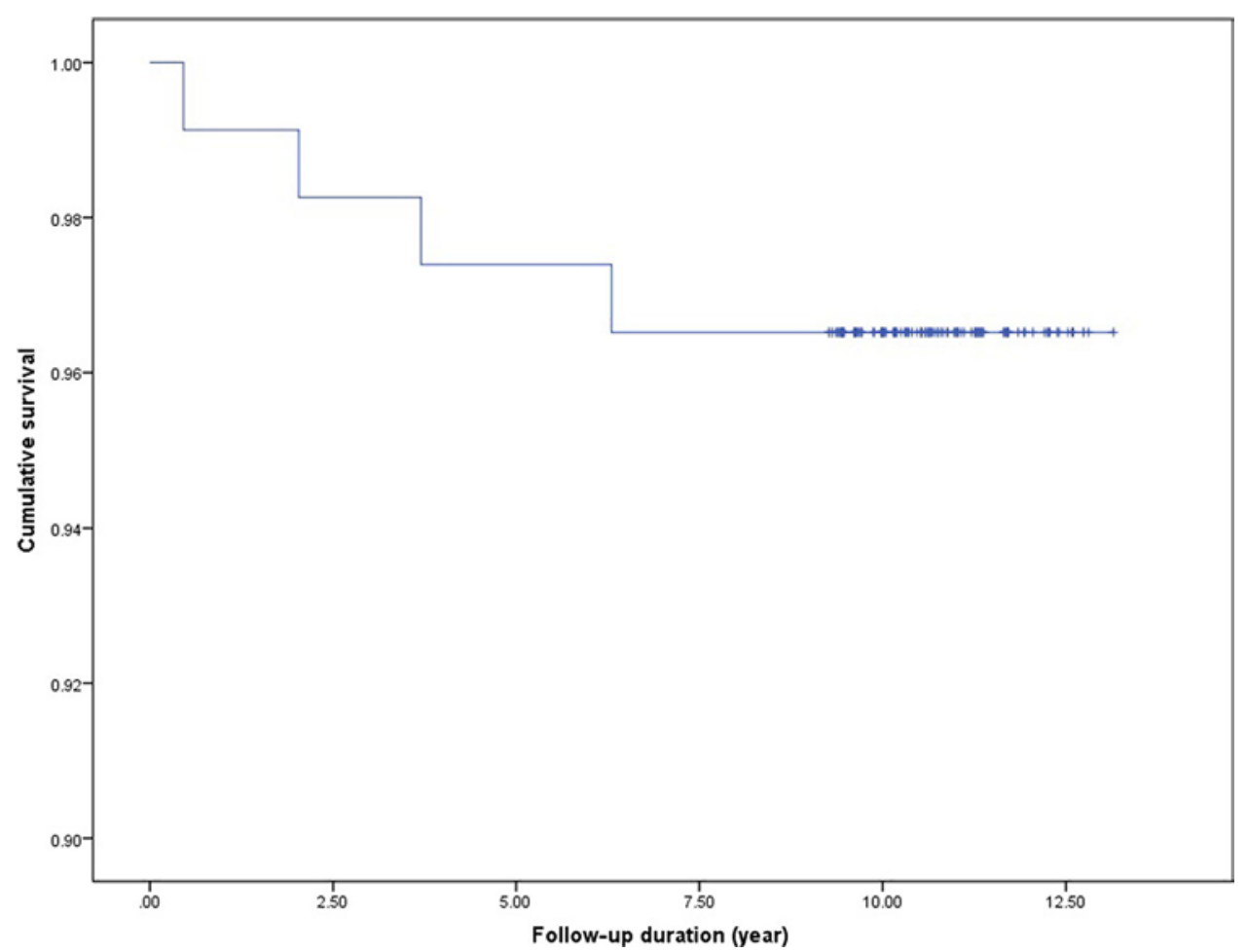

FIG. 3. Kaplan-Meier plot for cumulative survival after surgery in pediatric patients treated using surgical revascularization. Figure is available in color online only.

Multivariate regression analysis also demonstrated relationships between correlated factors and clinical outcomes $(\mathrm{F}=5.89, \mathrm{p}=0.00028)$. Those with an older age at symptom onset and the absence of preoperative stroke had better clinical outcomes $(b=0.067, p=0.0006 ; b=$ $-0.541, \mathrm{p}=0.001)$. Delay time and Suzuki stage did not significantly affect outcomes $(b=0.047, p=0.077 ; b=$ $-0.003, \mathrm{p}=0.96$; Table 3).

\section{Discussion}

Various operative procedures have been used for the treatment of pediatric MMD. However, the most effective method remains unclear, and there is no standard treatment. ${ }^{30}$ EDAS causes little trauma, and is a simple operation that maximally preserves the existing vascular network. In this study, we found that most pediatric patients achieved good recovery after EDAS, with no recurrent acute cerebrovascular events during the follow-up period. The results of follow-up angiography confirmed that EDAS had induced the formation of a new arterial network and compensatory CBF. This study also reported on a longer follow-up in a larger sample and provided data on EDAS in the treatment of pediatric MMD.

Our cohort mainly presented with symptoms of cerebral ischemia. The peak age at onset was about 10 years, and familial MMD occurrence was $11.3 \%$. These characteristics were similar to those in Japanese and South Korean reports; ; $^{12,15,19,21,26,37,41}$ however, the female/male ratio was different from those in Japanese and South Korean cohorts but similar to that in a Chinese cohort. ${ }^{18,36}$ The dif- ference may be related to ethnicity as there was no difference in the sex distribution of MMD in China. Our large cohort underwent long-term follow-up. Of concern, $63.4 \%$ of patients experienced a treatment delay $>1$ year, and the average period ( 2.82 years) was longer than that in the previous 5 years (1.97 years). Moreover, a large number of patients were initially misdiagnosed and treated incorrectly, indicating a lack of knowledge about the diagnosis and treatment of MMD by primary medical care providers. A review of the literature yielded few data, so we present our findings as reflective of the diagnosis and treatment of MMD in China. Although there was no statistically significant correlation between delay time and preoperative neurological function $(\mathrm{p}=0.846)$, we found that some patients with mild symptoms had a longer delay. There was also no significant correlation between delay time and

TABLE 3. Multivariate regression analysis of correlated factors and clinical outcomes

\begin{tabular}{lcl}
\hline \multicolumn{1}{c}{ Characteristic } & $b$ & $p$ Value \\
\hline Age at symptom onset & 0.067 & $\mathbf{0 . 0 0 0 6}$ \\
\hline Delay time & 0.047 & 0.077 \\
\hline Preop stroke & -0.541 & $\mathbf{0 . 0 0 1}$ \\
\hline Suzuki stage & -0.003 & 0.96 \\
\hline
\end{tabular}

Multivariate regression analysis demonstrated relationships between correlated factors and clinical outcomes (adjusted $R^{2}=0.165, F=5.89, p=$ 0.00028). Boldface type indicates statistical significance. 
clinical outcome. Rather, outcome was mainly related to the natural course of MMD. It is possible that flow volume and compliance were greater in these patients due to cerebrovascular reserve capacity. Nonetheless, $10.44 \%$ of patients in our series had stroke events because of a delay in treatment.

In our series, $92 \%$ of patients had an improved or unchanged mRS score. Kuroda et al. ${ }^{29}$ reported a 3.2\% annual incidence of stroke in symptom-free MMD children, and $37 \%$ exhibited neurological deficits in the absence of surgical treatment. In our series, the 10-year cumulative risk of recurrent stroke was 3.5\%, and the 5-year risk $(2.6 \%)$ was lower than the rates $(5.8 \%-15 \%)$ reported by other groups. 7,8,16,33,39 This suggests that EDAS is effective for pediatric MMD and can improve local cerebral hemodynamics, increase cerebral blood supply, reduce the risk of stroke, and improve neurological function. Longterm outcomes were better in patients without infarction and with lower Suzuki stages. There was no correlation with extensive collateral circulation. Thus, we believe that the extent of compensation depended on the degree of cerebral ischemia, and that recovery was limited by permanent neurological damage. Surgical treatment can prevent and reduce the risk of stroke recurrence and can improve the prognosis, survival status, and quality of life. In children, these effects are more significant with timely improvement in cerebral perfusion, which promotes brain development and remodeling of brain function after stroke. However, in children younger than 3 years, the risk of stroke was higher than that in older children. In our series, 2 children had acute cerebral infarctions and cried loudly after surgery, and crying probably led to an unstable hemodynamic status. Therefore, better perioperative care is essential.

Various studies have reported complications after revascularization surgery for MMD, and it is generally recognized that perioperative complications are important contributors to outcome. ${ }^{28}$ The incidence of postoperative complications was $3 \%$ in 232 procedures, but 2 patients had permanent neurological deficits. These results were similar to or better than the $1.6 \%-16.0 \%$ reported by other groups, whether for EDAS or other revasculariza-

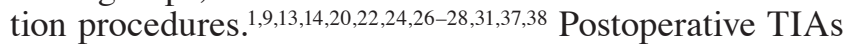
or infarctions of various sizes were the most clinically relevant complications observed in our department. Possible important contributory factors were unstable hemodynamic status and significantly impaired cerebrovascular reserve capacity in patients with an advanced Suzuki stage. Perioperative care should include adequate hydration, but antiplatelet agents are contraindicated in pediatric patients.

Our results showed that EDAS provides excellent angiographic and clinical results in pediatric patients, consistent with data reported in the literature. ${ }^{23,38}$ Researchers have assumed that the potential for blood vessel repair was better in younger patients than in older patient groups and adults. ${ }^{2,3,17,25,32}$ However, our results showed no significant correlation between collateral circulation and age at symptom onset. Moreover, despite longer delay times and preoperative infarction causing severe cerebral hypoperfusion, abundant collateral circulation developed in these pa- tients. These results indicated that the extent of collateral circulation was mainly related to the degree of cerebral ischemia; the worse the CBF, the better the collateral circulation.

In this study, we evaluated long-term angiographic responses and clinical outcomes following surgical treatment of MMD in Chinese children. EDAS is safe and effective for pediatric patients with MMD and can reduce the risk of subsequent neurological events and improve quality of life. The risk of ischemia-related complications was higher in younger patients, and older children showed better outcomes. Compensation was greater with more prominent cerebral ischemia. Long-term outcomes largely depended on the presence or extent of cerebral infarction.

\section{Study Limitations}

This was a retrospective study and thus had some limitations. EDAS is a preferred procedure and comprises $\geq$ $90 \%$ of the revascularization operations performed at our institute. We did not compare different surgical treatments as the disparity in sample size was too large, and no significant data set could be generated. Thus, larger samples are needed and longer follow-up should be performed to identify better treatments for MMD. Because the effect of MMD on cognitive function was not evaluated, improvement after revascularization was not studied. Moreover, improvements in cerebral hemodynamics and cerebrovascular reserve were not evaluated because of different imaging methods.

Much is still unknown about the pathogenesis of MMD and the factors influencing postoperative prognosis. In addition, many health care providers lack adequate knowledge about MMD. Outcomes for patients with MMD may benefit from advances in science and technology along with further research on diagnosis and treatment.

\section{Conclusions}

On the basis of long-term follow-up data, we concluded that EDAS is a safe and effective treatment for pediatric MMD, can reduce the risk of subsequent neurological events, and can improve quality of life. The risk of ischemia-related complications was higher in younger patients, and older children showed better outcomes. Compensation was greater with more prominent cerebral ischemia. The long-term clinical outcome largely depended on the presence and extent of preoperative stroke.

\section{Acknowledgments}

We thank the individuals who contributed to the study or manuscript preparation but did not fulfill all the criteria of authorship.

This study was supported by grants from the National Nature Science Foundation of China (Grant Nos. 81571136 and 81601021) and Beijing Municipal Science \& Technology Commission (No. Z171100001017144).

\section{References}

1. Adelson PD, Scott RM: Pial synangiosis for moyamoya syndrome in children. Pediatr Neurosurg 23:26-33, 1995

2. Arias EJ, Dunn GP, Washington CW, Derdeyn CP, Chicoine MR, Grubb RL Jr, et al: Surgical revascularization in North 
American adults with moyamoya phenomenon: long-term angiographic follow-up. J Stroke Cerebrovasc Dis 24:15971608,2015

3. Asfora WT, West M, McClarty B: Angiography of encephalomyosynangiosis and superficial temporal artery to middle cerebral artery anastomosis in moyamoya disease. AJNR Am J Neuroradiol 14:29-30, 1993

4. Baltsavias G, Khan N, Valavanis A: The collateral circulation in pediatric moyamoya disease. Childs Nerv Syst 31:389398, 2015

5. Bamford JM, Sandercock PA, Warlow CP, Slattery J: Interobserver agreement for the assessment of handicap in stroke patients. Stroke 20:828, 1989

6. Bao XY, Duan L, Yang WZ, Li DS, Sun WJ, Zhang ZS, et al: Clinical features, surgical treatment, and long-term outcome in pediatric patients with moyamoya disease in China. Cerebrovasc Dis 39:75-81, 2015

7. Cheng JH, Ye Q, Ye ZS, Li ZQ, Xu AD: Five-year experience of 101 adult patients with moyamoya disease at a single institution in Eastern China. J Clin Neurosci 32:30-34, 2016

8. Chiu D, Shedden P, Bratina P, Grotta JC: Clinical features of moyamoya disease in the United States. Stroke 29:13471351, 1998

9. Choi JU, Kim DS, Kim EY, Lee KC: Natural history of moyamoya disease: comparison of activity of daily living in surgery and non surgery groups. Clin Neurol Neurosurg 99 (Suppl 2):S11-S18, 1997

10. Digiusto M, Bhalla T, Grondin R, Tobias JD: Perioperative care of the pediatric patient for pial synangiosis surgery. Int J Clin Exp Med 6:231-238, 2013

11. Duan L, Bao XY, Yang WZ, Shi WC, Li DS, Zhang ZS, et al: Moyamoya disease in China: its clinical features and outcomes. Stroke 43:56-60, 2012

12. Fukui M, Kono S, Sueishi K, Ikezaki K: Moyamoya disease. Neuropathology 20 Suppl:S61-S64, 2000

13. Funaki T, Takahashi JC, Takagi Y, Yoshida K, Araki Y, Kikuchi T, et al: Incidence of late cerebrovascular events after direct bypass among children with moyamoya disease: a descriptive longitudinal study at a single center. Acta Neurochir (Wien) 156:551-559, 2014

14. Goda M, Isono M, Ishii K, Kamida T, Abe T, Kobayashi H: Long-term effects of indirect bypass surgery on collateral vessel formation in pediatric moyamoya disease. $\mathbf{J}$ Neurosurg 100 (2 Suppl Pediatrics):156-162, 2004

15. Han DH, Kwon OK, Byun BJ, Choi BY, Choi CW, Choi JU, et al: A co-operative study: clinical characteristics of 334 Korean patients with moyamoya disease treated at neurosurgical institutes (1976-1994). Acta Neurochir (Wien) 142:1263-1274, 2000

16. Hishikawa T, Tokunaga K, Sugiu K, Date I: Long-term outcomes in adult patients with ischemic-type moyamoya disease involving posterior circulation. Acta Neurochir (Wien) 156:1745-1751, 2014

17. Houkin K, Kuroda S, Ishikawa T, Abe H: Neovascularization (angiogenesis) after revascularization in moyamoya disease. Which technique is most useful for moyamoya disease? Acta Neurochir (Wien) 142:269-276, 2000

18. Hung CC, Tu YK, Su CF, Lin LS, Shih CJ: Epidemiological study of moyamoya disease in Taiwan. Clin Neurol Neurosurg 99 (Suppl 2):S23-S25, 1997

19. Ikezaki K, Han DH, Kawano T, Kinukawa N, Fukui M: A clinical comparison of definite moyamoya disease between South Korea and Japan. Stroke 28:2513-2517, 1997

20. Imai H, Miyawaki S, Ono H, Nakatomi H, Yoshimoto Y, Saito N: The importance of encephalo-myo-synangiosis in surgical revascularization strategies for moyamoya disease in children and adults. World Neurosurg 83:691-699, 2015

21. Imaizumi T, Hayashi K, Saito K, Osawa M, Fukuyama
Y: Long-term outcomes of pediatric moyamoya disease monitored to adulthood. Pediatr Neurol 18:321-325, 1998

22. Ishikawa T, Houkin K, Kamiyama H, Abe H: Effects of surgical revascularization on outcome of patients with pediatric moyamoya disease. Stroke 28:1170-1173, 1997

23. Isono M, Ishii K, Kamida T, Inoue R, Fujiki M, Kobayashi H: Long-term outcomes of pediatric moyamoya disease treated by encephalo-duro-arterio-synangiosis. Pediatr Neurosurg 36:14-21, 2002

24. Karasawa J, Touho H, Ohnishi H, Miyamoto S, Kikuchi H: Long-term follow-up study after extracranial-intracranial bypass surgery for anterior circulation ischemia in childhood moyamoya disease. J Neurosurg 77:84-89, 1992

25. Kawaguchi S, Okuno S, Sakaki T: Effect of direct arterial bypass on the prevention of future stroke in patients with the hemorrhagic variety of moyamoya disease. J Neurosurg 93:397-401, 2000

26. Kim SK, Cho BK, Phi JH, Lee JY, Chae JH, Kim KJ, et al: Pediatric moyamoya disease: an analysis of 410 consecutive cases. Ann Neurol 68:92-101, 2010

27. Kim T, Oh CW, Bang JS, Kim JE, Cho WS: Moyamoya disease: treatment and outcomes. J Stroke 18:21-30, 2016

28. Kim T, Oh CW, Kwon OK, Hwang G, Kim JE, Kang HS, et al: Stroke prevention by direct revascularization for patients with adult-onset moyamoya disease presenting with ischemia. J Neurosurg 124:1788-1793, 2016

29. Kuroda S, Hashimoto N, Yoshimoto T, Iwasaki Y; Research Committee on Moyamoya Disease in Japan: Radiological findings, clinical course, and outcome in asymptomatic moyamoya disease: results of multicenter survey in Japan. Stroke 38:1430-1435, 2007

30. Kuroda S, Houkin K: Moyamoya disease: current concepts and future perspectives. Lancet Neurol 7:1056-1066, 2008

31. Kuroda S, Houkin K, Ishikawa T, Nakayama N, Iwasaki Y: Novel bypass surgery for moyamoya disease using pericranial flap: its impacts on cerebral hemodynamics and long-term outcome. Neurosurgery 66:1093-1101, 2010

32. Macyszyn L, Attiah M, Ma TS, Ali Z, Faught R, Hossain $A$, et al: Direct versus indirect revascularization procedures for moyamoya disease: a comparative effectiveness study. J Neurosurg 126:1523-1529, 2017

33. Mallory GW, Bower RS, Nwojo ME, Taussky P, Wetjen NM, Varzoni TC, et al: Surgical outcomes and predictors of stroke in a North American white and African American moyamoya population. Neurosurgery 73:984-991, 981-982, 2013

34. Matsushima T, Inoue T, Suzuki SO, Fujii K, Fukui M, Hasuo $\mathrm{K}$ : Surgical treatment of moyamoya disease in pediatric patients-comparison between the results of indirect and direct revascularization procedures. Neurosurgery 31:401405, 1992

35. Matsushima Y, Fukai N, Tanaka K, Tsuruoka S, Inaba Y, Aoyagi M, et al: A new surgical treatment of moyamoya disease in children: a preliminary report. Surg Neurol 15:313-320, 1981

36. Miao W, Zhao PL, Zhang YS, Liu HY, Chang Y, Ma J, et al: Epidemiological and clinical features of moyamoya disease in Nanjing, China. Clin Neurol Neurosurg 112:199-203, 2010

37. Mukawa M, Nariai T, Matsushima Y, Tanaka Y, Inaji M, Maehara T, et al: Long-term follow-up of surgically treated juvenile patients with moyamoya disease. J Neurosurg Pediatr 10:451-456, 2012

38. Scott RM, Smith JL, Robertson RL, Madsen JR, Soriano SG, Rockoff MA: Long-term outcome in children with moyamoya syndrome after cranial revascularization by pial synangiosis. J Neurosurg 100 (2 Suppl Pediatrics):142-149, 2004

39. Starke RM, Komotar RJ, Hickman ZL, Paz YE, Pugliese AG, 
Otten ML, et al: Clinical features, surgical treatment, and long-term outcome in adult patients with moyamoya disease. Clinical article. J Neurosurg 111:936-942, 2009

40. Suzuki J, Takaku A: Cerebrovascular "moyamoya" disease. Disease showing abnormal net-like vessels in base of brain. Arch Neurol 20:288-299, 1969

41. Takanashi J: Moyamoya disease in children. Brain Dev 33:229-234, 2011

42. Wang KC, Phi JH, Lee JY, Kim SK, Cho BK: Indirect revascularization surgery for moyamoya disease in children and its special considerations. Korean J Pediatr 55:408-413, 2012

\section{Disclosures}

The authors report no conflict of interest concerning the materi- als or methods used in this study or the findings specified in this paper.

\section{Author Contributions}

Conception and design: Duan, Y Zhang, Bao. Acquisition of data: Y Zhang, Bao. Analysis and interpretation of data: Y Zhang, Bao. Drafting the article: Y Zhang, Bao. Critically revising the article: Duan, Y Zhang, Bao. Reviewed submitted version of manuscript: Duan, Y Zhang, Bao. Approved the final version of the manuscript on behalf of all authors: Duan. Statistical analysis: Y Zhang, Yang, Li, ZS Zhang, Han, Zhao, Q Zhang, Wang. Administrative/technical/material support: Yang, Li, ZS Zhang, Han, Zhao, Q Zhang, Wang. Study supervision: Duan.

\section{Correspondence}

Lian Duan: 307th Hospital of People's Liberation Army, Beijing, People's Republic of China. duanlian307@sina.com. 